\title{
CULTURAL METHOD IN BABESIOSIS PATHOGENS DIAGNOSIS: CURRENT STATE OF THE PROBLEM
}

DOI: 10.36740/WLek202105129

\author{
Inna I. Torianyk \\ STATE INSTITUTION «MECHNIKOV INSTITUTE OF MICROBIOLOGY AND IMMUNOLOGY OF THE NATIONAL ACADEMY OF MEDICAL SCIENCES OF \\ UKRAINE», KHARKIV, UKRAINE
}

\begin{abstract}
The aim is to show the effectiveness of the cultural method in the diagnosis of babesiosis.

Materials and methods: The study material was the blood from 10 healthy humans and animals, 30 humans with borreliosis, 41 animals with babesiosis. A cultural research method was used.

Results: The use of cultural method contributed to the optimization of the etiopathogenetic diagnosis of babesiosis. The method objectively proved the ability of protozoa (Babesia spp.) to asexual reproduction in vitro on the multicomponent growth medium with erythrocytes; proved the role of erythrocytes as acceptable for Babesia spp. of target cells. Absolute and relative parameters of detection of Babesia spp. by cultural method in the blood samples of the groups of the examined persons/species were different, making on average $19.7 \%$ (with the highest rates among the domestic dogs (62.5\%) and cattle (20.0\%)). Negative result (0\%) was identified among the patients with borreliosis.

Conclusions: Detection of Babesia spp. by the cultural method objectively confirmed its value as an apparent laboratory criterion for the etiopathogenetic diagnosis of babesiosis.
\end{abstract}

KEY WORDS: cultural method, babesiosis, diagnosis

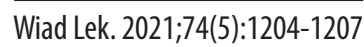

\section{INTRODUCTION}

Babesiosis is a typical zoonotic, emerging disease caused by a tick-borne intraerythrocytic protozoan of Babesia spp. that also can be transmitted by blood transfusion. Babesiosis imposes an increasing public-health threat [1]. Parasites of the genus Babesia infect a wide variety of domestic and wild mammals as well as man $[2,3]$.

In humans the disease manifestations are broad, from asymptomatic through mild flu-like infection to severe malaria-like disease with a potentially fatal outcome, mainly in immunodeficient and/or elderly individuals [4].

Babesiosis is not easy to diagnose and a high index of suspicion is required, especially in endemic regions [5]. Improvement and expansion of methods for babesiosis diagnosis is an urgent problem of modern medicine.

\section{THE AIM}

The aim is to show the effectiveness of the cultural method in the diagnosis of babesiosis.

\section{MATERIALS AND METHODS}

The main methodological principles of babesiosis diagnosis using cultural methods were similar to the principles, on which microbiological methods of diagnosis of most infectious diseases were based and due to which the isolation of pathogen culture remained the «gold standard» of etiopathogenetic diagnosis of infections [6]. For the cultivation of Babesia spp. in vitro the most effective technology of prolonged cultivation of parasites in microaerophilic conditions of a stationary layer of erythrocytes (static phase) at the bottom of cultural containers was used. This technology is currently called «MASP» (microaerophylous stationary phase) [7].

The study material was the blood from humans and animals $(\mathrm{n}=71)$ (table 1$)$.

The multicomponent growth medium based on the RPMI-1640 (Roswell Park Memorial Institute 1640) medium (BioTestLab, Ukraine) was used for growing of Babesia spp. based on the blood samples of patients, cattle. The composition of the latter included $10 \%$ of serum of cattle (BioloT Ltd., Russian Federation), gentamicin (80 $\mu \mathrm{g} / \mathrm{ml}$ ) (Pharmaceutical company «Zdorov'ya», Ukraine), amphotericin B (50 $\mu \mathrm{g} / \mathrm{ml})$ (Open Joint-Stock Company Kurgan Medicines and Products «Sintez», Russian Federation), HEPES (25 mmol) (Serva-Feinbiochemia heldelberg, USA), sodium bicarbonate ( $25 \mathrm{mmol}$ ) (Lubnipharm, Ukraine). The final $\mathrm{pH}$ of the medium was 7.2-7.4. $10 \%$ of the serum of dogs and horses was added to the growth mediums for the cultivation of Babesia spp. on the basis of blood samples from dogs and horses. The growth mediums was filtered (pore diameter $0.22 \mu \mathrm{m}$ ), packed in sterile labeled containers, stored for two weeks at temperature $4 \pm 0.5^{\circ} \mathrm{C}$. Inoculations of suspensions of babesia-infected erythrocytes (infected red blood cells - iRBC) were put 
Table 1. The analysis of the study material

\begin{tabular}{cc}
\hline Name of objects & Quantity (in absolute and relative (\%) indices) \\
\hline Patients with borreliosis & $30(42.3 \%)$ \\
\hline Cattle with babesiosis & $20(28.2 \%)$ \\
\hline Horses with babesiosis & $5(7.0 \%)$ \\
\hline Bitten by ticks domestic dogs with babesiosis & $16(22.5 \%)$ \\
\hline
\end{tabular}

Table 2. The results of the detection of Babesia spp.

\begin{tabular}{cccc}
\hline \multirow{2}{*}{ Name of the object } & \multicolumn{3}{c}{ Number of positive results in absolute and relative (\%) indices } \\
\cline { 2 - 4 } & Cultural method & Microscopic method & $\begin{array}{c}\text { Polymerase chain reaction standard } \\
\text { format with primers Bab system }\end{array}$ \\
\hline Patients with borreliosis & 0 & 0 & 0 \\
\hline Cattle & $4(20.0 \%)$ & $8(40.0 \%)$ & $8(40.0 \%)$ \\
\hline Horses & 0 & $5(100 \%)$ & $5(100 \%)$ \\
\hline Domestic dogs & $10(62.5 \%)$ & $11(68.8 \%)$ & $11(68.8 \%)$ \\
\hline Total & $14(19.7 \%)$ & $24(33.8 \%)$ & $24(33.8 \%)$ \\
\hline
\end{tabular}

in flat-bottom culture plates (Cellstar corporation, USA; Realab, Ukraine) and the growth mediums (with a ratio of their volumes in the system 1:10). The contents of the later were incubated for three weeks at temperature $37 \pm 0.5^{\circ} \mathrm{C}$ in a humidified atmosphere with $5 \%$ carbon dioxide $\left(\mathrm{CO}_{2}\right.$, desiccator conditions) providing in vitro microaerophilic conditions for culturing a static layer of erythrocytes under the growth mediums. The total volume of the well suspension (iRBC + growth medium, iRBC + growth medium + $\mathrm{RBC}$ ) ranged from $2.0 \mathrm{ml}$ to $2.2 \mathrm{ml}$. Inoculations of $\mathrm{iRBC}$ from all the studied blood samples from humans, cattle, horses, dogs were made in three parallel (experimental) wells. The same volume of RBC (suspensions of uninfected autologous erythrocytes) was added to the control wells instead of iRBC. In one well of the culture plate (and in each series of experimental studies) only growth medium for cultivation was put (without addition of any erythrocytes) to control the sterility. During the incubation period the growth medium was replaced daily (sometimes in a day), and every five to seven days, together with the growth medium replacement, the inoculations (cultures of Babesia $s p p$. in iRBC) were fed by uninfected RBCs.

The suspensions of uninfected autologous $\mathrm{RBC}$ of homologous species were used to feed in vitro the cultures initiated by iRBC. The sources of production of sterile (according to the results of previous studies) for babesia erythrocyte suspensions were volunteer donors $(4,40 \%)$, cattle $(2,20 \%)$, horse $(1,10 \%)$, domestic dogs $(3,30 \%)$.

The level of parasitaemia was monitored from the third to the seventh day from the beginning of incubation and performed daily (sometimes every other day). The percentage of infected erythrocytes was calculated by examining at least a thousand erythrocytes (target cells that were most often affected by Babesia spp.).

Reaching the level of parasitaemia in the culture about 5-7\%, after replacement of the growth medium reinoculation was made (subsequent passages) in new wells in the ratio of the volume of cell suspension from the previous "old well and the volume of the prepared growth medium with RBC 1:2, respectively. Subsequent subcultures of $B a$ besia spp. were grown at a ratio of the volume of the «old» culture to the volume of the growth medium with RBC for cultivation 1:4, respectively.

Verification of the affiliation of in vitro grown hemoparasites to the genus Babesia and identification of species ( $B$. microti, $B$. divergens and $B$. canis) were performed using the microscopic method and polymerase chain reaction.

\section{RESULTS AND DISCUSSION}

The studies of the blood samples of volunteer donors showed an absolutely negative result. In any of the cases the confirmation of the presence of Babesia spp. was not found. Assumptions about the possible reproduction of babesiosis pathogens in the blood samples from patients with borreliosis (as a comorbid disease for babesiosis) were groundless.

The level of detection of Babesia spp. by the cultural method resources in the blood samples was $19.7 \%$. The quantitative structure was built on the results of the surveys of domestic dogs and cattle. The vast majority of positive detection results were the blood samples taken from domestic dogs (62.5\%) (table 2). The second position was held the blood samples taken from cattle $(20.0 \%)$.

However, the obtained parameters in this study were lower $(\mathrm{p}<0.05)$ compared to similar indicators in the cases of using microscopic method and polymerase chain reaction standard format with primers Bab system (table 2). The negative result in the blood samples from horses was explained by the specifics of the cultural material, which prevented the growth of parasites, despite the positive results in the cases of using microscopic method and polymerase chain reaction standard format with primers Bab system. 
Conduction of the cultural method investigation in this study suggested us about the mandatory use of additional methods for the detection of Babesia spp. in biological material favorable to the mentioned parasitosis warm-blooded animals. The latter fact showed the demand for objective verification of the results obtained under the conditions of cultural method (taking into account the species differences of Babesia spp. and detection of new/understudied parasites of this family).

On the other hand, the adjudicated data substantiated the necessity of practical implementation of the cultural methods. They contributed to the objective production of strains (domestic dogs with clinical manifestations of babesiosis), including B. canis (additional polymerase chain reaction identification: with a positive result using primer systems Bab and Bac, and negative one - Bdi and Bmi). The established level of effectiveness of the cultural method was determined by a number of factors recognized by the professional schools in Europe and the world [8].

The technological logistics of cultural method approbation demonstrated an obvious duration of the study (equal to or greater than 21 days), exceptional quantity of professional skills, complexity of the technology of this method reproduction. These factors were preconditioned by the demand for time-constant monitoring of the cultivation of Babesia spp. in iRBC, replacement of the growth medium, feeding with uninfected RBC. The performance of sequential passages of parasite cultures in order to maintain their viability and permissiveness was time-consuming.

The evident general tendency of gradual increase (reproduction) of Babesia spp. in the suspensions of cultured erythrocytes (during the first ten days from the beginning of incubation) did not give clear staging and synchronization of the processes. Deficiencies found in the conditions of implementation, testing of cultural method were associated with the lysis of cultured erythrocyte suspensions, which caused the cessation of parasite reproduction, reduced effective visualization of pathogens. The «reverse» dynamics of the invasive process in vitro correlated with the dynamics of lysis (death) of erythrocyte cultures, where Babesia spp. supported their own vital functions. The known factors of lysis of erythrocyte cultures microsporous fungi Micromonospora purpurea/Micromonospora echinospora (gentamicin producers) had a negative impact on the procedural links of cultural method [9].

The latter fact led to the use of antibiotic-containing media such as RPMI-1640 for prevent bacterial germination [10]. The deficiency was eliminated by selecting antibacterial, antifungal drugs (Moxifloxacin hydrochloride (moxifloxacin) manufactured by Nosch Labs Private Ltd. (India) and Fluconasole (fluconazole) manufactured by Vitalife laboratories (Apollo)). The effective concentration of moxifloxacin and fluconazole in the initial solutions (prepared with sterile distilled water for injection) was 2.0 $\mathrm{mg} / \mathrm{ml}$. Preparation of multicomponent growth medium for the cultivation of erythrocyte suspensions involved an addition of each of the initial solutions of antimicrobial components ( $100.0 \mu \mathrm{l}$ per $100.0 \mathrm{ml}$ of the growth medium) to achieve the final concentration of moxifloxacin and fluconazole of $2 \mu \mathrm{g} / \mathrm{ml}$. The use of multicomponent growth medium in the cultivation of samples of $\mathrm{R} R \mathrm{BC}$ and $\mathrm{RBC}$, showed that the addition of moxifloxacin and fluconazole to the medium content objectively prevented the germination of bacteria and fungi for 21 days, toxic effects on the erythrocyte suspension, prevented the inhibitory effect targeted to the reproduction and permissiveness of cultures of Babesia spp.

\section{CONCLUSIONS}

The use of cultural method contributed to the optimization of the etiopathogenetic diagnosis of babesiosis. The method objectively proved the ability of protozoa (Babesia $s p p$.) to asexual reproduction in vitro on the multicomponent growth medium with erythrocytes; proved the role of erythrocytes as acceptable for Babesia spp. of target cells. Absolute and relative parameters of detection of Babesia spp. by cultural method in the blood samples of the groups of the examined persons/species were different, making on average $19.7 \%$ (with the highest rates among the domestic dogs $(62.5 \%)$ and cattle (20.0\%)). Negative result (0\%) was identified among the patients with borreliosis.

\section{REFERENCES}

1. Zhou X, Xia S, Huang JL, Tambo E, Zhuge HX, Zhou XN. Human babesiosis, an emerging tick-borne disease in the people's Republic of China. Parasit Vectors. 2014;7:509. doi: 10.1186/s13071-014-0509-3.

2. Mosqueda J, Olvera-Ramirez A, Aguilar-Tipacamu G, Canto GJ. Current advances in detection and treatment of babesiosis. Current Medicinal Chemistry. 2012;19(10):1504-1518.

3. Beugnet $F$, Moreau Y. Babesiosis. Revue scientifique et technique. 2015;34(2):627-639.

4. Ord RL, Lobo CA. Human babesiosis: pathogens, prevalence, diagnosis and treatment. Current Clinical Microbiology Reports. 2015;2(4):173-181.

5. Parija SC, Kp D, Venugopal H. Diagnosis and management of human babesiosis. Tropical Parasitology. 2015;5(2):88-93.

6. Voroshilina ES, Zornikov DL, Panacheva EA. Sravnitelnoe issledovanie mikrobioty jejakuljata metodom kolichestvennoj PCR i kulturalnym metodom. Evaluation of the ejaculate microbiota by real-time PCR and culture-based technique. Bulletin of Russian State Medical University. 2019;1:44-49. (Ru).

7. Álvarez Martínez JA, Figueroa Millán JV, Ueti MW, Rojas-Martínez C. Innovative alternatives for continuous in vitro culture of Babesia bigemina in medium free of components of animal origin. Pathogens. 2020;9(5):343. doi: 10.3390/pathogens9050343

8. Riley PA. Principles of microscopy, culture and serology-based diagnostics. Diagnosis of infection. 2013;41(11):658-662.

9. Wei Z, Shi X, Lian R, Wang W, Hong W, Guo S. Exclusive production of gentamicin C1a from Micromonospora purpurea by metabolic engineering. Antibiotics (Basel). 2019;8(4):267. doi:10.3390/ antibiotics8040267

10. Ihalamulla RL, Siriwardana HV, Karunaweera ND. Efficacy of RPMI 1640 and M 199 media in the isolation of Leishmania from cutaneous lesions. Annals of Tropical Medicine and Parasitology. 2008;102(2):173-175. 
ORCID and contributionship:

Inna I. Torianyk: 0000-0001-6843-8808 A,B,C,D,E,F

\section{Conflict of interest:}

The Author declare no conflict of interest.

\section{CORRESPONDING AUTHOR}

Inna I. Torianyk

Laboratory of Viral Infections, State Institution

«Mechnikov Institute of Microbiology and Immunology

of the National Academy of Medical Sciences of Ukraine»

39 Kamysheva Ivana str., apt. 9, 61038 Kharkiv, Ukraine

e-mail: patholognew@ukr.net

Received: 13.11.2020

Accepted: 02.04.2021

A - Work concept and design, B - Data collection and analysis, C - Responsibility for statistical analysis,

$\mathbf{D}$-Writing the article, $\mathbf{E}$-Critical review, $\mathbf{F}$ - Final approval of the article 